\title{
Self-consistent description of proton radioactivity
}

\author{
Lidia Ferreira* \\ Centro de Física das Interacções Fundamentais, \\ and Departmento de Física, Instituto Superior Técnico, Lisbon \\ E-mail: Elidiadist.ut1.pt
}

\section{Enrico Maglione}

Dipartimento di Fisica "G. Galilei”, Via Marzolo 8, I-35131 Padova, Italy and Istituto Nazionale di Fisica Nucleare, Padova, Italy

\section{Peter Ring}

Physik-Department der Technischen Universität München, D-85748 Garching, Germany

Proton emission from spherical nuclei lying beyond the proton drip-line is described by a fully self-consistent calculation based on relativistic density functionals derived from meson exchange and point coupling models.

50th International Winter Meeting on Nuclear Physics

23-27 January 2012

Bormio, Italy

\footnotetext{
* Speaker.
} 


\section{Introduction}

With modern experimental facilities [ $[$ ] nuclei with proton or neutron excess are now becoming accessible in an attempt to map the limits of stability and determine the nuclear properties away from the stability valley. The extraordinary development of trapping techniques, the use of storage rings and new detection procedures, have provided high precision measurements of masses, and decay observables which form a basic testing ground for the nuclear structure models and underlying fundamental theories. Whereas on the neutron rich side, exotic nuclei with neutron skins and halos []] were found, on the proton rich side, nuclei that could spontaneously decay by emitting one [B]] or two protons [四, 回], were found beyond the drip line. Proton radioactivity was then the process that signalized the proton drip line in the region of nuclear charges between $\mathrm{Z}=50$ and $\mathrm{Z}=83$, and at lower charges where emission of two protons occurs.

Quite a few proton radioactive nuclei were found until now, decaying from the ground or from an excited state to the ground or excited states of the daughter nucleus. Thus, data gathered from fine structure, complemented by the decay widths and spectra built on the decaying states, obtained from decay tagging, constitute a valuable set of information on the structure of the nuclei involved in the process. In fact, the decay properties are highly sensitive [6-O] to details of the nuclear wave function. If the theory is able to account for the data, it will be able to assign the spin and parity of the decaying state, and the nuclear shape. Proton emitters can display spherical symmetry but some present axial or even non- axial deformation, results hinted by the experimental data and confirmed theoretically[ए], [1]. Proton emission has been thoroughly studied in non relativistic models based on phenomenological mean field descriptions of the nucleus, obtained by fitting the single particle properties of stable nuclei. For details, see Ref.[[2] and references therein.

Recent microscopic models, based on covariant density functional theory (CDFT) have been quite successful in the description of nuclear structure properties of stable nuclei, and at the limits of stability[[13]]. Time-dependent versions of this theory have been successfully used for the description of excited states such as rotational bands or giant resonances. When compared with non-relativistic density functionals, covariant density functional theory has some advantages, since it accounts in a natural way for the spin-orbit interaction, without the need of any extra adjustable parameters, and to a natural saturation mechanism of the force, due to the subtle compensation between the large attractive scalar and the large repulsive vector fields. Chiral symmetry and pseudospin symmetry are also incorporated in the theory. Therefore, it needs only a relatively small number of parameters that are adjusted to reproduce a set of bulk properties of finite nuclei, and nuclear matter, and it is valid over the entire periodic table. The proton drip-line was mapped with high precision [14-[16], within the relativistic Hartree-Bogoliubov (RHB) theory [17, [8] for spherical and deformed nuclei, and the single particle configurations and spectroscopic factors, successfully derived. However, all these applications of RHB theory have been carried out by expanding the single particle Dirac spinors in a basis of harmonic oscillator wave functions. A drawback of this method is the poor treatment of the tails of the single particle wave functions, and consequently it is not applicable to study decay processes, where a correct description of the decaying state is crucial. In order to overcome this difficulty, and study nuclear decay by one proton emission, we performed a fully self-consistent relativistic calculation, where the differential Dirac equation was solved with outgoing wave boundary conditions. The interaction used was based on 
relativistic density functionals derived from meson exchange and point coupling models, which we will discuss below.

\section{Covariant density functional theory for nuclei}

Relativistic versions of nuclear density functional theory [[13, [19-2]] start from a covariant Lagrangian, where the nucleus is described as a system of interacting Dirac particles. In order to keep relativistic invariance and causality the interaction has to be mediated either by the exchange of mesons or it has to be of zero range. In this investigations we compare results obtained with two of these models, the non-linear meson exchange model NL3 [22] and the density dependent point coupling model DD-PC1 [리].

\subsection{Non-linear meson exchange models}

The standard representation of covariant density functional theory uses the Walecka model of Quantum Hadrodynamics as a vehicle to introduce Lorentz invariance. The nucleons are described by the Dirac spinors. They interact in an effective Lagrangian through the exchange of mesons and the electromagnetic field.

According to their quantum numbers of spin and isospin, the isoscalar scalar $\sigma$-meson, the isoscalar vector $\omega$-meson, and the isovector vector $\rho$-meson build a minimal set of meson fields that together with the electromagnetic field is necessary for a quantitative description of bulk and single-particle properties in nuclei [19, 201, 24]. This Lagrangian has the form

$$
\mathscr{L}=\mathscr{L}_{N}+\mathscr{L}_{m}+\mathscr{L}_{\text {int }},
$$

where $\mathscr{L}_{N}$ denotes the Lagrangian of free nucleons with the mass $m$

$$
\mathscr{L}_{N}=\bar{\psi}\left(i \gamma^{\mu} \partial_{\mu}-m\right) \psi
$$

$\mathscr{L}_{m}$ is the Lagrangian of free meson fields and the electromagnetic field

$$
\begin{aligned}
\mathscr{L}_{m} & =\frac{1}{2} \partial_{\mu} \sigma \partial^{\mu} \sigma-\frac{1}{2} m_{\sigma}^{2} \sigma^{2}-\frac{1}{4} \Omega_{\mu v} \Omega^{\mu v}+\frac{1}{2} m_{\omega}^{2} \omega_{\mu} \omega^{\mu} \\
& -\frac{1}{4} \vec{R}_{\mu v} \vec{R}^{\mu v}+\frac{1}{2} m_{\rho}^{2} \vec{\rho}_{\mu} \vec{\rho}^{\mu}-\frac{1}{4} F_{\mu v} F^{\mu v}
\end{aligned}
$$

with the corresponding masses $m_{\sigma}, m_{\omega}, m_{\rho}$, and the field tensors

$$
\begin{aligned}
\Omega_{\mu v} & =\partial_{\mu} \omega_{v}-\partial_{v} \omega_{\mu}, \\
\vec{R}_{\mu v} & =\partial_{\mu} \vec{\rho}_{v}-\partial_{v} \vec{\rho}_{\mu}, \\
F_{\mu v} & =\partial_{\mu} A_{v}-\partial_{v} A_{\mu} .
\end{aligned}
$$

Here arrows denote isovectors. $\mathscr{L}_{\text {int }}$ contains the minimal set of covariant interactions

$$
\begin{aligned}
\mathscr{L}_{\text {int }} & =-g_{\sigma} \bar{\psi} \sigma \psi-g_{\omega} \bar{\psi} \gamma_{\mu} \omega^{\mu} \psi \\
& -g_{\rho} \bar{\psi} \gamma_{\mu} \vec{\tau} \vec{\rho}^{\mu} \psi-e \bar{\psi} \gamma_{\mu} A^{\mu} \psi
\end{aligned}
$$




\begin{tabular}{|c|c|c|c|c|c|c|c|}
\hline$m$ & $=939$ & & & & & & \\
\hline$m_{\sigma}$ & $=508.194$ & $g_{\sigma}$ & $=10.217$ & $g_{2}$ & $=-10.431$ & $g_{3}$ & $=-28.885$ \\
\hline$m_{\omega}$ & $=782.501$ & $g_{\omega}$ & $=12.868$ & & & & \\
\hline$m_{\rho}$ & $=763.000$ & $g_{\rho}$ & $=4.474$ & & & & \\
\hline
\end{tabular}

Table 1: Parameters of the effective interaction NL3. The masses are given in units of MeV, $g_{2}$ is in units of $\mathrm{fm}^{-1}$ and the remaining parameters are dimensionless

with the coupling constants $g_{\sigma}, g_{\omega}, g_{\rho}$ and the electromagnetic charge $e$ which vanishes for neutrons.

This simple model, with interaction terms linear in the meson fields, does, however, not provide a quantitative description of the nuclear surface properties. An effective density dependence was introduced [25] by replacing the quadratic $\sigma$-potential $\frac{1}{2} m_{\sigma}^{2} \sigma^{2}$ with a quartic potential

$$
U(\sigma)=\frac{1}{2} m_{\sigma}^{2} \sigma^{2}+\frac{g_{2}}{3} \sigma^{3}+\frac{g_{3}}{4} \sigma^{4} .
$$

This potential includes non-linear $\sigma$ self-interactions and introduces two additional parameters $g_{2}$ and $g_{3}$. This particular form of the potential has become standard in applications of RMF models. Here we use the successful parameter set NL3. Its parameters are given in Table $\mathrm{W}$.

From the Lagrangian density the classical variation principle leads to the equations of motion. In the static case considered in this investigation we obtain for the nucleons the Dirac equation which is equivalent to the Kohn-Sham equations [26] in non-relativistic density functional theory

$$
[\alpha p+V+\beta(m+S)] \psi=0 .
$$

It contains a scalar and a vector potential

$$
\begin{aligned}
S(r) & =g_{\sigma} \sigma(r), \\
V(r) & =g_{\omega} \omega(r)+g_{\rho} \tau_{3} \rho_{3}(r)+e A_{0}(r),
\end{aligned}
$$

where $\omega(r)$ and $\rho_{3}(r)$ are the time-like components of the vector meson fields. The meson fields obey the Klein-Gordon equations

$$
\begin{aligned}
\left(-\Delta+m_{\sigma}^{2}\right) \sigma & =-g_{\sigma} \rho_{s}-g_{2} \sigma^{2}-g_{3} \sigma^{3} \\
\left(-\Delta+m_{\omega}^{2}\right) \omega & =g_{\omega}\left(\rho_{p}+\rho_{n}\right) \\
\left(-\Delta+m_{\rho}^{2}\right) \rho_{3} & =g_{\rho}\left(\rho_{n}-\rho_{n}\right) \\
-\Delta A_{0} & =e \rho_{p} .
\end{aligned}
$$

The sources are formed by the scalar and vector densities

$$
\rho_{s}=\sum_{k} \bar{\psi}_{k} \psi_{k} v_{k}^{2}, \text { and } \rho_{\tau}=\sum_{k, \tau_{k}=\tau} \psi_{k}^{\dagger} \psi_{k} v_{k}^{2}
$$


where the index $\tau=p, n$ represents protons and neutrons and $v_{k}^{2}$ are occupation numbers for the various orbits. Pairing correlations are taken into account in the constant gap approximation

$$
v_{k}^{2}=\frac{1}{2}\left[1-\frac{\left(\varepsilon_{k}-\lambda\right)}{\sqrt{\left(\varepsilon_{k}-\lambda\right)^{2}+\Delta^{2}}}\right]
$$

with the empirical value for the gap parameter [DZ]

$$
\Delta=12 / A^{1 / 2}
$$

Starting with an initial guess for the potentials of Woods-Saxon form this non-linear set of equations is solved by iteration and the results are the potentials $S(r)$ and $V(r)$, which are used in a second step in the calculation of proton emitters and their decay probabilities.

RMF models can be also formulated without explicitly including meson-exchange interactions but replacing them by local four-point interactions between the nucleons [28]. A density dependence is introduced either by additional local six- and eight-point interactions [29] or by density dependent coupling constants [23], which are equivalent procedures at the Hartree mean field level.

In the present work, we will also use the recent parameter set DD-PC1 [23], derived from a high precision fit to nuclear matter data and to the binding energies of 64 deformed nuclei in the rare earth and actinide region. The potential has been quite successful in the description of exotic nuclei, and it has a density dependence given by

$$
\alpha_{i}(\rho)=a_{i}+\left(b_{i}+c_{i} x\right) e^{-d_{i} x}
$$

The quantity $x=\rho / \rho_{\text {sat }}$ is the density in units of the saturation density of nuclear matter $\rho_{\text {sat }}=$ $0.152 \mathrm{fm}^{-3}$ and the parameters $a_{i}, b_{i}, c_{i}$, and $d_{i}$ are given in Table $\square$.

Variations on the Lagrangean obtained in this formulation lead to the scalar and vector potentials

$$
\begin{aligned}
S & =\alpha_{S} \rho_{s}-\delta_{S} \Delta \rho_{s} \\
V & =\alpha_{V} \rho+\alpha_{T V} \rho_{3}+e A_{0}
\end{aligned}
$$

and a rearrangement term in the isoscalar vector channel

$$
R=\frac{1}{2}\left\{\alpha_{S}^{\prime} \rho_{s}^{2}+\alpha_{V}^{\prime} \rho^{2}+\alpha_{T V}^{\prime} \rho_{3}^{2}\right\}
$$

arising from the variation of the vertex functionals $\alpha_{S}(\rho), \alpha_{V}(\rho)$, and $\alpha_{T V}(\rho)$ with respect to the nucleon fields in the density operator: $\alpha_{i}^{\prime}=d \alpha_{i} / d \rho$. It is essential for energy-momentum conservation and the thermodynamical consistency of the model. For details, see Ref. [ए2]].

\subsection{Self-consistent description of spherical proton emitters}

The main characteristic of proton emitters relies upon their existence beyond the drip line, therefore the proton is a resonance in the nuclear mean field. Since the proton escapes with an energy of $1-2 \mathrm{MeV}$, these resonances lie very low in the continuum, and are essentially single particle resonances. The non-linear meson exchange model NL3 and the density dependent point coupling 


\begin{tabular}{lccccc}
\hline \hline$i$ & $a_{i}$ & $b_{i}$ & $c_{i}$ & $d_{i}$ & $\delta_{S}$ \\
$\mathrm{~S}$ & -10.0462 & -9.1504 & -6.4273 & -1.3724 & -0.8149 \\
$\mathrm{~V}$ & 5.9195 & 8.8637 & 0 & 0.6584 & \\
$\mathrm{TV}$ & 0 & 1.8360 & 0 & 0.6403 & \\
\hline \hline
\end{tabular}

Table 2: The coupling constants of the parameter set DD-PC1. The parameters $a_{i}, b_{i}$, and $c_{i}$ are given in units of $\mathrm{fm}^{-2}$, the parameter $d_{i}$ is dimensionless and the parameter $\delta_{S}$ is given in units of $\mathrm{fm}^{-4}$

model DD-PC1 were then applied to the calculation of single particle resonances in spherical nuclei drip-line nuclei. According to scattering theory, the half-life for decay by one particle emission is given by $T_{1 / 2}=\hbar \ln 2 / \Gamma$, where the decay width can be found [目] from the relation:

$$
\Gamma=S \frac{\hbar^{2} k \alpha^{2}}{m}
$$

with $m$ and $k$ standing for the mass and wave number of the proton. $S$ is a spectroscopic factor, and the quantity $\alpha$ the asymptotic normalization of the proton wave function. The latter is a solution of the Dirac equation with outgoing wave boundary conditions in the spherical NL3 and DD-PC1 fields. In order to obtain the resonance at the experimental energy, the depth of the nuclear potential was slightly changed, whereas the Coulomb potential was kept unchanged. The spectroscopic factor in the case of nuclei where the residual interaction is mainly due to pairing, is simply the probability $u_{k}^{2}$ that the single particle level $k$ is empty in the daughter nucleus, and can be calculated in the BCS approach assuming the pairing gap of Eq. (2.J7).

Following this procedure, all spherical proton emitters that have been observed, were calculated, and a comparison with the experimental results shows a very good qualitative agreement. See for example table II of Ref.[ए2]. However, since the half-lives ranges from $3 \mu$ s up to $10 \mathrm{~s}$, it is interesting to analyze the deviation from the experimental results.

In Fig. 四, we present the results for the half-life for decay from the h11/2 single particle state. The ratio between the theoretical and experimental half-lives as a function of the atomic number shows that close to the magic number 82 , for protons or neutrons, that is, in the vicinity of ${ }^{155} \mathrm{Ta}$ the agreement is very good for both NL3 and DD-PC1 interactions. Going away from this half-magicity, the ratio slowly decreases. The reason for this behavior can be attributed to the correlations in the nuclear medium, when nuclei are far from a closed shell. In such cases there will be a strong mixing of wave functions and a coupling to phonon states. In addition, for open proton and neutron shells, deformations will set in. The spectroscopic factor will become smaller than the one derived from the BCS model, since pairing is not the only dominating effective interaction in this case. Therefore, the discrepancy between theory and experiment must increase as one is moving away from magic numbers. In fact, recent observation of the spectra of some of these emitters has confirmed the role of triaxial deformation in ${ }^{161} \mathrm{Re}$ [四, B⿺] and ${ }^{145} \mathrm{Tm}$ [ [3]]. Theoretical studies [U] have confirmed that, with a quite large triaxial $\gamma$ deformation in ${ }^{145} \mathrm{Tm}$, the experimental energy spectra of parent and daughter nuclei, the half-life and the fine structure could be simultaneously interpreted. 


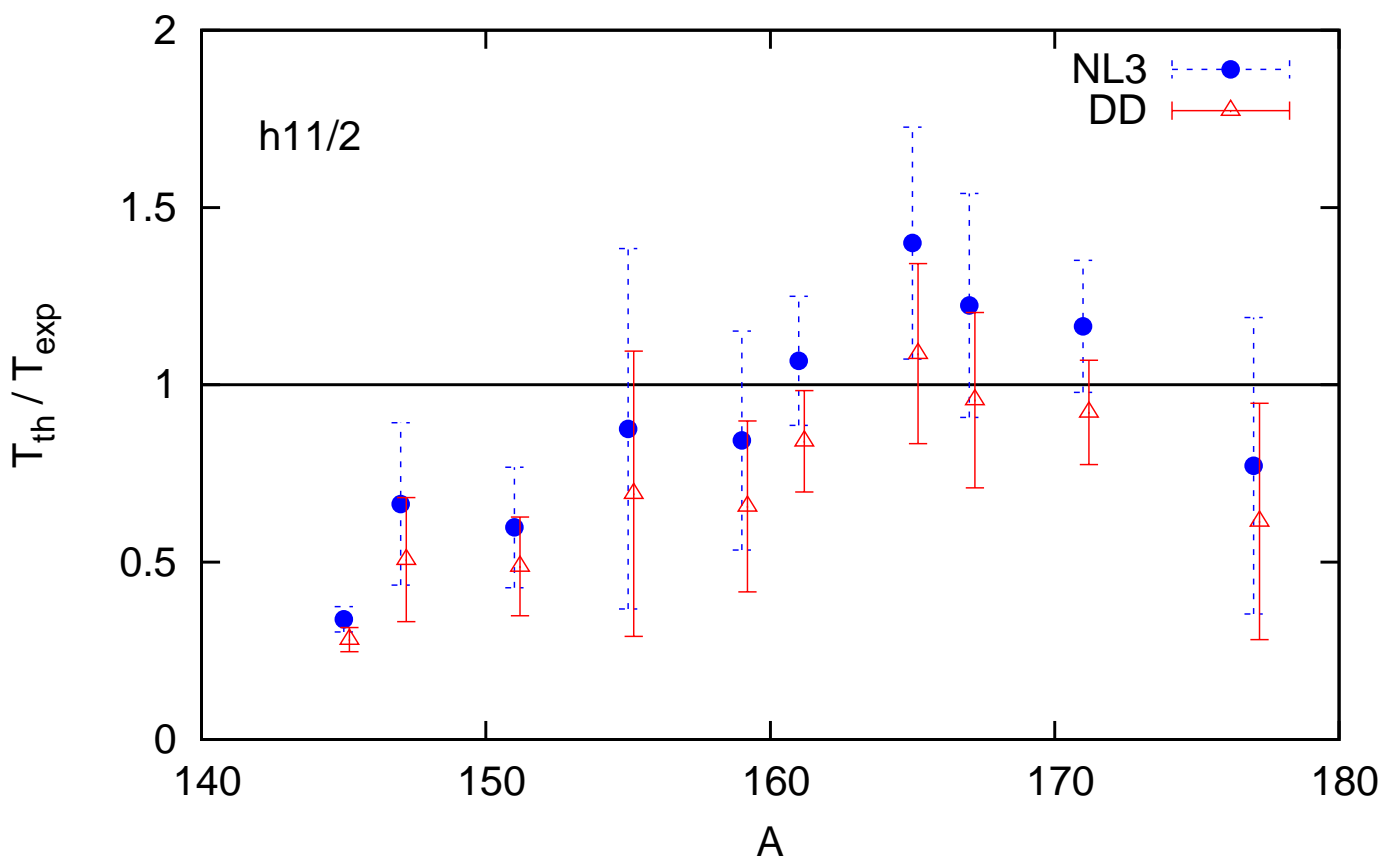

Figure 1: Ratio between the theoretical and experimental half-lives as a function of the mass number for decay from the h11/2 single particle level, using the NL3 (dot) and DD-PC1 fields (triangles). The error bars take into account the experimental error on the half-life, and the theoretical error induced by the experimental error on the energy.

Similar conclusion can be drawn when decay occurs from other single particle states, as discussed in Ref.[एٓ]].

In conclusion, using interactions derived from relativistic density functional theory, we have presented the first fully self-consistent model without free parameters, that accounts for the experimental data of proton radioactivity from spherical nuclei. The calculation has shown clear evidence of configuration mixing away from half magicity for proton or neutrons, and provided a new tests of the non-linear meson exchange NL3 [22] and density dependent point coupling DD-PC1 [23] models, at the extremes of nuclear stability.

\section{Acknowledgments}

This work was supported by the Fundação para a Ciência e a Tecnologia (Portugal), Project: CERN/FP/116385/2010 and by the DFG cluster of excellence "Origin and Structure of the Universe" (www.universe-cluster.de)

\section{References}

[1] NuPECC Long Range Plan 2010

[2] P. G. Hansen, A. S. Jensen, B. Jonson, Annu. Rev. Nucl. Phys. 45 (1995) 591.

[3] A. A. Sonzogni, Nucl. Data Sheets 95 (2002) 1. 
[4] M. Pfutzner, et al., Eur. Phys. J. A 14 (2002) 279.

[5] J. Giovinazzo, et al., Phys. Rev. Lett. 89 (2002) 102501.

[6] E. Maglione, L. S. Ferreira, R. J. Liotta, Phys. Rev. Lett. 81 (1998) 538.

[7] E. Maglione, L. S. Ferreira, R. J. Liotta, Phys. Rev. C 59 (1999) R589.

[8] L. S. Ferreira, E. Maglione, Phys. Rev. Lett. 86 (2001) 1721.

[9] G. Fiorin, E. Maglione, L. S. Ferreira, Phys. Rev. C 67 (2003) 054302.

[10] P. Arumugam, E. Maglione, L. S. Ferreira, Phys. Rev. C 76 (2007) 044311.

[11] P. Arumugam, L. S. Ferreira, E. Maglione, Phys. Rev. C 78 (2008) 041305.

[12] L. S. Ferreira, E. Maglione, P. Ring, Phys. Lett. B 701 (2011) 508.

[13] D. Vretenar, A. V. Afanasjev, G. A. Lalazissis, P. Ring, Phys. Rep. 409 (2005) 101.

[14] G. A. Lalazissis, D. Vretenar, P. Ring, Nucl. Phys. A 650 (1999) 133.

[15] G. A. Lalazissis, D. Vretenar, P. Ring, Nucl. Phys. A 679 (2001) 481.

[16] G. A. Lalazissis, D. Vretenar, P. Ring, Phys. Rev. C 69 (2004) 017301.

[17] D. Vretenar, G. A. Lalazissis, P. Ring, Phys. Rev. Lett. 82 (1999) 4595.

[18] G. A. Lalazissis, D. Vretenar, P. Ring, Phys. Rev. C 60 (1999) 051302(R).

[19] B. D. Serot, J. D. Walecka, Adv. Nucl. Phys. 16 (1986) 1.

[20] P. Ring, Prog. Part. Nucl. Phys. 37 (1996) 193.

[21] J. Meng, H. Toki, S.-G. Zhou, S.-Q. Zhang, W.-H. Long, L.-S. Geng, Prog. Part. Nucl. Phys. 57 (2006) 470 .

[22] G. A. Lalazissis, J. König, P. Ring, Phys. Rev. C 55 (1997) 540.

[23] T. Nikšić, D. Vretenar, P. Ring, Phys. Rev. C 78 (2008) 034318.

[24] P.-G. Reinhard, Rep. Prog. Phys. 52 (1989) 439.

[25] J. Boguta, A. R. Bodmer, Nucl. Phys. A 292 (1977) 413.

[26] W. Kohn, L. J. Sham, Phys. Rev. 137 (1965) A1697.

[27] A. Bohr, B. R. Mottelson, Nuclear Structure Vol. 1, Benjamin, New York 1969.

[28] P. Manakos, T. Mannel, Z. Phys. A 334 (1989) 481.

[29] T. Bürvenich, D. G. Madland, J. A. Maruhn, P.-G. Reinhard, Phys. Rev. C 65 (2002) 044308.

[30] K. Lagergren, et al., Phys. Rev. C 74 (2006) 024316.

[31] D. Seweryniak, et al., Phys. Rev. Lett. 99 (2007) 082502. 